\title{
Research on Initial Alignment of All-Attitude Inertial Platform on Static Base
}

\author{
Hongjie Zhang ${ }^{1,}$, Shifeng Zhang ${ }^{1, b}$ \\ ${ }^{1}$ College of Aerospace Science and Engineering, National University of Defense Technology, \\ Changsha 410073, China \\ ahitzhanghongjie@163.com, byang_shifeng@hotmail.com
}

\begin{abstract}
In view of the current problem that long alignment time and low alignment accuracy in traditional alignment methods, a new two-step alignment method is proposed in this paper. This method consists of the coarse and accurate alignment. The feature of gravity vector is used to carry out the analytic coarse alignment. And by applying torque to the platform, the accurate alignment is carried out with extended Kalman filter (EKF). Simulation results show that the estimation error of the azimuthal misalignment angle can converge to less than 20 " in eight minutes.
\end{abstract}

Keywords: platform inertial navigation system, inertial alignment, analytic alignment, closed-loop EKF.

\section{Introduction}

Initial alignment means obtaining a direction cosine matrix between the geographic frame and the platform frame [1] before the inertial navigation system (INS) getting into work [2]. The alignment accuracy and time have a vital impact on the accuracy and reaction time of the inertial navigation system on strategic weapons.

A lot of researches about initial alignment have been done by scholars at home and abroad. To improve the alignment accuracy, Cao [3] proposed a rapid alignment method by using continuous spinning of the inertial platform. In Ref. [4], Kim studied the rotatory north-finding scheme. And the deviation caused by the gyro bias can be eliminated within one cycle. To avoid the problem of the poor observability of INS on stationary base, Fang [5] calculated the azimuth error directly on the basis of the fast convergence of the leveling error. As a result, the initial alignment time is greatly reduced. Ren [6] proposed a fast alignment method which introduced a torque feedback loop. The outputs of gyros were used to solve the azimuth. And by using this proposed method, the azimuth error can reach in 8 minutes. Although many scholars used the filter to complete the initial alignment $[7,8,9]$, few of them tried the closed-loop filter. The difference of the work in this paper lies in the use of the accelerometer outputs at a series of different time to perform the coarse alignment and the error compensation in the EKF circle.

In this paper, the analytical alignment is carried out which takes advantage of the feature of gravity vector. The analytical alignment can reduce the misalignment angles to meet the small misalignment assumption. Then the EKF algorithm is used to carry out the accurate alignment by applying torque to rotate the platform.

\section{Coarse Alignment of Stationary Inertial Platform}

The analytical alignment which is double-vector attitude determination essentially [10] is used as the coarse alignment in this paper. The direction cosine matrix between the geographic frame and the platform frame can be calculated by the conical motion of the gravity vector. And then, the initial coarse alignment is completed. 


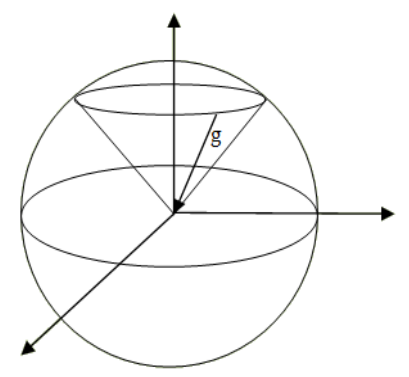

Fig. 1 Conical motion of gravity somewhere on the earth

\subsection{Coordinate Systems}

Coordinate systems needed in later sections are defined as follows:

Geocentric frame (e-frame): the origin Oe is the geometrical center of the earth. The OeXe axis lies in the equatorial plane and points to the prime meridian. The OeZe axis is along the earth's axis. The OeYe axis complements the right-handed system. This coordinate system is relative stationary to the ground and rotates with the earth.

Earth centered inertial frame (i-frame): the e-frame at initial time is chosen as the i-frame. It is noteworthy that the i-frame is stationary relative to the inertial space.

Platform frame (p-frame): its origin Op is the geometrical center of the platform. The OpXp axis is parallel to the input axis of the X-accelerometer. The OpYp axis lies in the plane determined by the input axes of the X-accelerometer and Y-accelerometer. And the OpYp axis is perpendicular to the OpXp axis.

Platform inertial frame (ip0-frame): the initial p-frame is chosen as the ip0-frame. Note that the ip0-frame is stationary relative to the inertial space.

Calculating frame (c-frame): this frame is a digital frame established by the navigation computer. The c-frame is not completely coincident with the p-frame because of the misalignment angles which are caused by the gyro drift and installation error etc.

Navigation frame (n-frame): take the present local frame as the n-frame which is the N-U-E frame.

Accelerometer sensitivity axes frame (sa-frame): the axes of the sa-frame are respectively parallel with the three input axes of the accelerometer in the three directions.

Gyro sensitivity axes frame (sg-frame): the axes of the sg-frame are respectively parallel with the three input axes of the gyros in the three directions.

Assume that the geographical position of the platform is $(\lambda, L) . \lambda$ and $L$ represent the geographic longitude and latitude of the static base respectively. The attitude matrix of the platform can be written as

$$
C_{p}^{n}=C_{i}^{n} C_{p}^{i}
$$

where $\mathbf{C}_{p}^{n}$ is the direction cosine matrix from p-frame to n-frame.

The direction cosine matrix from i-frame to n-frame can be expressed as $\boldsymbol{C}_{i}^{n}=\boldsymbol{C}_{e}^{n} \boldsymbol{C}_{i}^{e}$. According to the position $(\lambda, L)$, the direction cosine matrix from e-frame to $n$-frame can be given by

$$
\boldsymbol{C}_{e}^{n}=\left[\begin{array}{ccc}
-\sin L \cos \lambda & -\sin L \sin \lambda & \cos L \\
\cos L \cos \lambda & \cos L \sin \lambda & \sin L \\
-\sin \lambda & \cos \lambda & 0
\end{array}\right]
$$

The $\boldsymbol{C}_{i}^{e}$ can be written as

$$
\boldsymbol{C}_{i}^{e}=\left[\begin{array}{ccc}
\cos \theta & \sin \theta & 0 \\
-\sin \theta & \cos \theta & 0 \\
0 & 0 & 1
\end{array}\right]
$$


where $\theta=\omega_{i e}\left(t_{k}-t_{0}\right)$, represents the angle that the earth turns from $t_{0}$ to $t_{k}$. According to Eqs.(2) and (3), the direction matrix form i-frame to $n$-frame can be obtained

$$
\boldsymbol{C}_{i}^{n}=\boldsymbol{C}_{e}^{n} \boldsymbol{C}_{i}^{e}
$$

According to Eq.(1), in order to solve $C_{p}^{n}$, the $C_{p}^{i}$ is needed to be solved at first. With the transformation relation among the $\mathrm{p}$-frame, $\mathrm{i}_{\mathrm{p} 0}$-frame and $\mathrm{i}$-frame, the direction cosine matrix from p-frame to i-frame $C_{p}^{i}$ can be calculated as

$$
C_{p}^{i}=C_{i_{p 0}}^{i} C_{p}^{i_{p 0}}
$$

The relation between the $\boldsymbol{C}_{p}^{i_{p 0}}$ and the absolute angular rates of the platform is shown as follows

$$
\dot{\boldsymbol{C}}_{p}^{i_{p 0}}=\boldsymbol{C}_{p}^{i_{p 0}}\left[\boldsymbol{\omega}_{i_{p 0} p}^{p} \times\right]
$$

where $\boldsymbol{\omega}_{i_{00} p}^{p}$ is the absolute angular rates of the platform.

Ignoring the gyro drift and the noise, the commanded angular rates of the platform and $\boldsymbol{\omega}_{i_{p 0} p}^{p}$ are related with the following equation

$$
\boldsymbol{\omega}_{i_{p 0} p}^{p} \approx \boldsymbol{\omega}_{c m d}^{p}
$$

$\boldsymbol{C}_{p}^{i_{p 0}}$ can be solved with Eqs. (6) and (7). Till now, refering to Eqs. (1) and (5), the direction cosine matrix $\boldsymbol{C}_{p}^{n}$ can be solved if only $\boldsymbol{C}_{i_{p 0}}^{i}$ is solved. The detail of the scheme to complete the coarse alignment is shown in the next section.

\subsection{The Coarse Alignment Scheme under Static Base.}

The output of the accelerometer on the platform can be expressed as

$$
Z_{a}=K_{a 0}+\left(K_{a 1}+I_{3}\right) a_{A I}+K_{a 1}^{*}\left|a_{A I}\right|+v
$$

where $\boldsymbol{K}_{a 0}$ is the accelerometer biases. $\boldsymbol{K}_{a 1}$ represents the scale factor error. $\boldsymbol{K}_{\boldsymbol{a} 1}^{*}$ is the asymmetry of the scale factor. $\boldsymbol{v}$ is the measurement noise. $\boldsymbol{a}_{\boldsymbol{A} \boldsymbol{I}}$ is the input of the accelerometer which can be written as

$$
\boldsymbol{a}_{A I}=\boldsymbol{C}_{p}^{s a} \boldsymbol{C}_{n}^{p}\left(-\boldsymbol{g}^{n}\right)
$$

where $\boldsymbol{g}^{n}$ is the gravity vector. Substituting Eq. (9) into Eq. (8) yields

$$
\begin{aligned}
& \boldsymbol{Z}_{a}=\boldsymbol{K}_{a 0}+\left(\boldsymbol{K}_{a 1} \boldsymbol{C}_{p}^{s a}+\boldsymbol{C}_{p}^{s a}\right) \boldsymbol{C}_{n}^{p}\left(-\boldsymbol{g}^{n}\right)+\boldsymbol{K}_{a 1}^{*}\left|\boldsymbol{a}_{A I}\right|+\boldsymbol{v} \\
& =\boldsymbol{C}_{n}^{p}\left(-\boldsymbol{g}^{n}\right)+\left[\boldsymbol{K}_{a 0}+\boldsymbol{K}_{a 1} \boldsymbol{C}_{p}^{s a} \boldsymbol{C}_{n}^{p}\left(-\boldsymbol{g}^{n}\right)+\boldsymbol{C}_{p}^{s a} \boldsymbol{C}_{n}^{p}\left(-\boldsymbol{g}^{n}\right)-\boldsymbol{C}_{n}^{p}\left(-\boldsymbol{g}^{n}\right)+\boldsymbol{K}_{a 1}^{*}\left|\boldsymbol{a}_{A I}\right|+\boldsymbol{v}\right]
\end{aligned}
$$

where $\nabla_{a}^{p}=\boldsymbol{K}_{a 0}+\boldsymbol{K}_{a 1} \boldsymbol{C}_{p}^{s a} \boldsymbol{C}_{n}^{p}\left(-\boldsymbol{g}^{n}\right)+\boldsymbol{C}_{p}^{s a} \boldsymbol{C}_{n}^{p}\left(-\boldsymbol{g}^{n}\right)-\boldsymbol{C}_{n}^{p}\left(-\boldsymbol{g}^{n}\right)+\boldsymbol{K}_{a 1}^{*}\left|\boldsymbol{a}_{A I}\right|+\boldsymbol{v}$. Then Eq. (10) transforms into

$$
\boldsymbol{Z}_{a}=\boldsymbol{C}_{n}^{p}\left(-\boldsymbol{g}^{n}\right)+\nabla_{a}^{p}
$$

Combining Eqs. (1), (5) and (11) yields

$$
\boldsymbol{C}_{i_{p 0}}^{i} \boldsymbol{C}_{p}^{i_{p 0}} \boldsymbol{Z}_{a}-\boldsymbol{C}_{n}^{i} \nabla_{a}^{i}=\boldsymbol{C}_{n}^{i}\left(-\boldsymbol{g}^{n}\right)
$$

where $\boldsymbol{Z}_{a}$ is the output of the accelerometer. $\boldsymbol{C}_{n}^{i}$ can be solved by Eq. (4). Therefore, the problem to solve the $C_{p}^{n}$ transforms into how to solve $C_{i_{p 0}}^{i}$. Ignoring the impact of $\nabla_{a}$, Eq. (12) can be written as

$$
\boldsymbol{C}_{i_{p 0}}^{i} \boldsymbol{Z}_{a}^{i_{p 0}}=\boldsymbol{C}_{n}^{i}\left(-\boldsymbol{g}^{n}\right)
$$

Rotating around the earth's axis, the gravity vector at any two different moments is non-collinear. Taking this feature into account, the following equations can be gotten 


$$
\left\{\begin{array}{l}
\boldsymbol{C}_{i_{p 0}}^{i} \boldsymbol{Z}_{a 1}^{i_{p 0}}=\boldsymbol{C}_{n 1}^{i}\left(-\boldsymbol{g}^{n 1}\right) \\
\boldsymbol{C}_{i_{p 0}}^{i} \boldsymbol{Z}_{a 2}^{i_{p 0}}=\boldsymbol{C}_{n 2}^{i}\left(-\boldsymbol{g}^{n 2}\right) \\
\boldsymbol{C}_{i_{p 0}}^{i}\left(\boldsymbol{Z}_{a 1}^{i_{p 0}} \times \boldsymbol{Z}_{a 2}^{i_{p 0}}\right)=\left[\boldsymbol{C}_{n 1}^{i}\left(-\boldsymbol{g}^{n 1}\right)\right] \times\left[\boldsymbol{C}_{n 2}^{i}\left(-\boldsymbol{g}^{n 2}\right)\right]
\end{array}\right.
$$

where $\boldsymbol{Z}_{a 1} 、 \boldsymbol{Z}_{a 2}$ represent the output of the accelerometer at two different moments. Defining $\boldsymbol{M}=\left[\begin{array}{lll}\boldsymbol{Z}_{a 1}^{i_{p 0}} & \boldsymbol{Z}_{a 2}^{i_{p 0}} & \boldsymbol{Z}_{a 1}^{i_{p 0}} \times \boldsymbol{Z}_{a 2}^{i_{p 0}}\end{array}\right], \quad \boldsymbol{N}=\left[\boldsymbol{C}_{n 1}^{i}\left(-\boldsymbol{g}^{n 1}\right) \quad \boldsymbol{C}_{n 2}^{i}\left(-\boldsymbol{g}^{n 2}\right) \quad \boldsymbol{C}_{n 1}^{i}\left(-\boldsymbol{g}^{n 1}\right)\right] \times\left[\boldsymbol{C}_{n 2}^{i}\left(-\boldsymbol{g}^{n 2}\right)\right]$. According to Eq. (14), the direction from $\mathrm{i}$-frame to $\mathrm{i}_{\mathrm{p} 0}$-frame can be expressed as

$$
\boldsymbol{C}_{i}^{i_{p 0}}=\boldsymbol{M} \bullet \boldsymbol{N}^{-1}
$$

Till now, the direction cosine matrix of the platform can be totally solved.

\section{Accurate Alignment of Stationary All-Attitude Inertial Platform}

Regard the final results of coarse alignment as the initial filtering value. The accurate alignment is introduced in the next sections.

\subsection{System Dynamics Model}

After coarse alignment, the misalignment angles are sufficiently small to meet the small misalignment assumption [11]. Referring to Ref. [12], the system model can be written as

$$
\dot{\boldsymbol{\varphi}}=-\left[\boldsymbol{\omega}_{c m d} \times\right] \boldsymbol{\varphi}+\left(\boldsymbol{C}_{s g}^{p}+\Delta \boldsymbol{K}_{G}-\boldsymbol{I}_{3}\right) \boldsymbol{\omega}_{c m d}+\boldsymbol{\varepsilon}+\boldsymbol{w}
$$

where $\boldsymbol{\omega}_{c m d}$ is the commanded angular rates of the platform, which is the same as $\boldsymbol{\omega}_{i p}^{p}$. $\boldsymbol{C}_{s g}^{p}$ is the installation error matrix. $\Delta \boldsymbol{K}_{G}$ is the error coefficient matrix of the torque. $\boldsymbol{\varepsilon}$ is the platform drift. $\boldsymbol{w}$ is the noise.

Taking the X-gyro as an example, the error model can be written as

$$
\varepsilon_{x}=k_{g 0 x}+\left(k_{g 11 x} \dot{W}_{x}+k_{g 12 x} \dot{W}_{y}+k_{g 13 x} \dot{W}_{z}\right)+k_{g 31 x} \dot{W}_{x} \dot{W}_{y}+k_{g 32 x} \dot{W}_{x} \dot{W}_{z}+k_{g 33 x} \dot{W}_{y} \dot{W}_{z}+n_{x}
$$

where $\varepsilon_{x}$ is the X-gyro static drift. $k_{g 0 x}$ is the gyro bias. $k_{g 11 x}, k_{g 12 x} 、 k_{g 13 x}$ are the first-order coefficients. $k_{g 21 x} 、 k_{g 22 x} 、 k_{g 23 x}$ are the cross term coefficients. $n_{x}$ is the zero mean Gaussian white noise. $\dot{W}_{x} 、 \dot{W}_{y} 、 \dot{W}_{z}$ are the specific force.

Take the misalignment angles, accelerometer bias and gyro bias into account in the simulation, the system model can be simplified as

$$
\dot{\boldsymbol{\varphi}}=-\left[\omega_{c m d} \times\right] \boldsymbol{\varphi}+\boldsymbol{\varepsilon}
$$

And the measurement equation can be simplified as [13]

$$
\boldsymbol{Z}_{\delta a}=[\boldsymbol{\varphi} \times] \boldsymbol{g}^{c}+\boldsymbol{K}_{a 0}
$$

Choosing the misalignment angle, gyro bias and accelerometer bias as the state variables, $\boldsymbol{X}=\left[\begin{array}{lll}\boldsymbol{\varphi} & \boldsymbol{D}_{\boldsymbol{F}} & \boldsymbol{K}_{\mathrm{a} 0}\end{array}\right]^{\mathrm{T}}$.

$$
\dot{\boldsymbol{X}}=f(\boldsymbol{X})=\left[\begin{array}{c}
-\left[\boldsymbol{\omega}_{c m d} \times\right] \boldsymbol{\varphi}+\boldsymbol{\varepsilon} \\
\boldsymbol{0}_{3 \times 1} \\
\boldsymbol{0}_{3 \times 1}
\end{array}\right]
$$

Eq. (20) represents the Kalman filtering state equation.

\subsection{Kalman filter}

In order to prevent the misalignment angles from getting larger as time goes on, the closed-loop EKF is applied in this paper. To make the c-frame and p-frame coincide with each other, every filter result is used to compensate the attitude of the platform. 


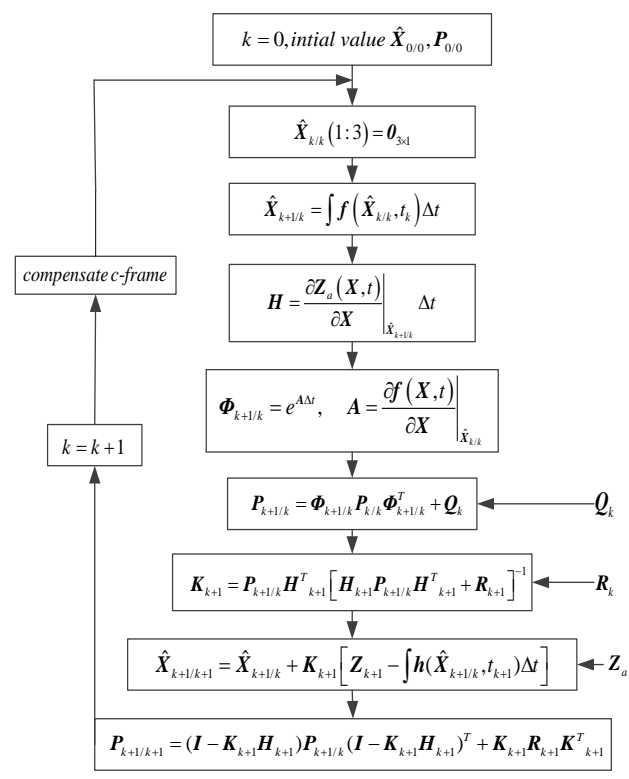

Fig. 2 Filter flow chart

Assume that $\hat{\boldsymbol{\varphi}}$ is the estimate of the misanlignment angle, the compensated $\boldsymbol{C}_{c}^{n}$ can be expressed as

$$
\hat{\boldsymbol{C}}_{c}^{n}=(\boldsymbol{I}-[\hat{\boldsymbol{\varphi}} \times]) \boldsymbol{C}_{c}^{n}
$$

\section{Simulation and Discussions}

\subsection{Simulation Parameters}

The level attitude error of the platform is uniform random distribution within $\pm 5^{\circ}$. The azimuth is uniform random distribution within $\pm 90^{\circ}$. The means and standard deviations of the rest state variables are shown in Table 1.

Table 1 the Mean and Standard Deviation of State Variables

\begin{tabular}{|c|c|c|c|}
\hline Parameter & Unit & Mean & Standard deviation \\
\hline$D_{F X}$ & ${ }^{\circ} / \mathrm{h}$ & 0 & $0.01 / 3$ \\
\hline$D_{F Y}$ & $\circ / \mathrm{h}$ & 0 & $0.01 / 3$ \\
\hline$D_{F Z}$ & $\circ / \mathrm{h}$ & 0 & $0.01 / 3$ \\
\hline$k_{a 0 x}$ & $\mu \mathrm{g}$ & 0 & $50 / 3$ \\
\hline$k_{a 0 y}$ & $\mu \mathrm{g}$ & 0 & $50 / 3$ \\
\hline$k_{a 0 z}$ & $\mu \mathrm{g}$ & 0 & $50 / 3$ \\
\hline
\end{tabular}

In the simulation, the period of applying torque is set to $0.1 \mathrm{~s}$. The filter period is set to $1 \mathrm{~s}$. The measurement noise of the accelerometer is set to $0.1 \mu \mathrm{g}$. The measurement noise of the gyro is set to $0.001^{\circ} / \mathrm{h}$. The inital value of misalignment angle is set as the result of the coarse alignment. And the rest inital values are all set to 0 .

\subsection{Simulation Results}

Using Monte Carlo method to simulate 1000 times, one of the results is as follows

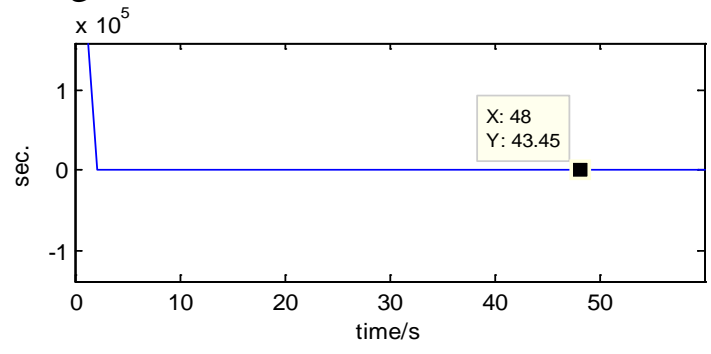

Fig. 3 Coarse alignment error of $\delta \varphi_{x}$

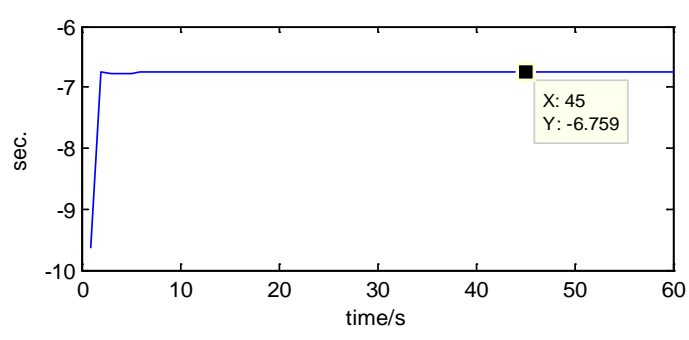

Fig. 4 Coarse alignment error of $\varphi_{y}$ 


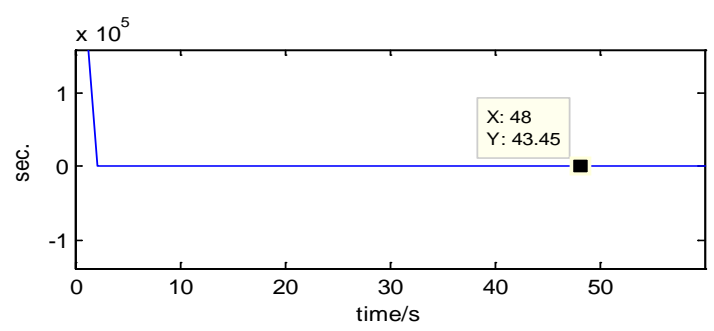

Fig. 5 Coarse alignment error of $\varphi_{z}$

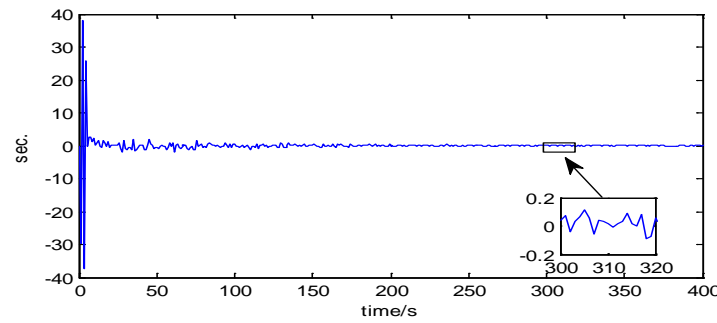

Fig. 7 Accurate alignment error of $\varphi_{y}$

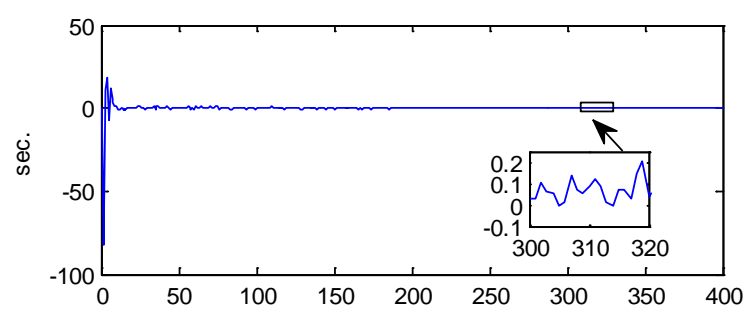

Fig. 6 Accurate alignment error of $\varphi_{x}$

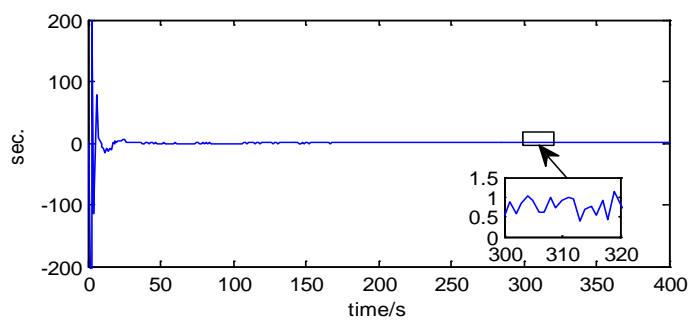

Fig. 8 Accurate alignment error of $\varphi_{z}$

where $\varphi_{x}$ and $\varphi_{y}$ represent the level attitude estimation error. $\varphi_{z}$ represents the azimuth estimation error.

The coarse alignment estimation error results are shown in Figs.3 5. It can be seen that the estimation errors can be reduced to small angles within 10 seconds. The small misalignment assumption is satisfied. The accurate results are shown in Figs.6 8. It can be seen that the estimation errors converge quickly. The total time is less than 8 minutes.

The Table 2 shows the mean values and standard deviations of 1000 times Monte Carlo simulation results.

Table 2 Simulation results

\begin{tabular}{|c|c|c|c|c|}
\hline \multirow[b]{2}{*}{ Parameter } & \multicolumn{2}{|c|}{ Coarse alignment } & \multicolumn{2}{|c|}{ Accurate alignment } \\
\hline & $\begin{array}{l}\text { Mean } \\
\text { (sec) }\end{array}$ & 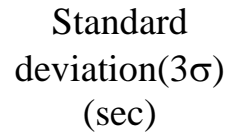 & $\begin{array}{c}\text { Mean } \\
\text { (sec) }\end{array}$ & $\begin{array}{c}\text { Standard } \\
\text { deviation }(3 \sigma) \\
\text { (sec) }\end{array}$ \\
\hline$\alpha$ & -0.0013 & 3.9384 & -0.0242 & 1.6406 \\
\hline$\beta$ & -1.1155 & 149.0133 & 0.0833 & 12.8112 \\
\hline$\gamma$ & -0.0501 & 4.2030 & -0.0327 & 1.3791 \\
\hline
\end{tabular}

It can be concluded that, after the coarse alignment, the estimation error of level attitude angle is less than $10 "$. The estimation error of azimuth is less than $200 "$. Then after the accurate alignment, the estimation errors of the level attitude angle and the azimuth become less than 5 " and 20 " respectively. The accurate alignment can obviously improve the alignment accuracy compared with the coarse alignment.

\section{Conclusions}

This paper presents a novel inertial alignment method consisting of coarse and accuracy alignment for all-attitude static base. The coarse alignment makes the misalignment angles sufficiently small in order to apply the accurate alignment. The accurate alignment makes the alignment error converge quickly. This proposed method shortens the alignment time and improves the alignment accuracy compared with the traditional method. The simulation results show that the azimuth alignment error can be up to within $20 "$ in 8 minutes. 


\section{References}

[1] C. Zhang, W. Tian and Z. Jin. A novel method improving the alignment accuracy of a strapdown inertial navigation system on a stationary base [J]. Measurement Science and Technology, 15 (2004) 765-769.

[2] G. Yan, J. Weng, L. Bai and Y. Qin. Inertial in-movement alignment and position determination based on inertial reference frame [J]. Systems Engineering and Electronics, 31(3) (2009) 634-637. (in Chinese)

[3] Y. Cao, S. Zhang, H. Yang and H. Cai. Fast self-alignment method for inertial platform through continuous rotation [J]. Acta Armamentarii, 32(12) (2011) 1468-1473. (in Chinese)

[4] S. J. Kim, S. S. Lee and Y. S. Kwon. Dynamic north-finding scheme based on a fiber gyroscope [C]. Proceedings of SPIE - The International Society for Optical Engineering, 1997.

[5] J. Fang, S. Zhu and W. Yu. Fast initial alignment method of inertial navigation system on stationary base [J]. Journal of Beijing University of Aeronautics and Astronautics, 25(6) (1999) 728-731. (in Chinese)

[6] H. Ren, Z. Tang. Fast alignment method for platform inertial navigation system [J]. Journal of Chinese Inertial Technology, 18(3) (2010) 279-282. (in Chinese)

[7] W. Gao, L. Miao and M, Ni. Multiple fading factors Kalman filter for SINS static alignment application [J]. Chinese Journal of Aeronautics, 24 (2011) 476-483.

[8] H. Dai, S. Zhou, M. Chen and X. Wu. Quaternion based on nonlinear error model for rapid transfer alignment [J]. Journal of Astronautics, 31(10) (2010) 2328-2334.

[9] J. Wang, J. Song, Z. Li and D. Chao. Application of time series modeling in initial alignment of strapdown fiber optic (FOG) compass system [J]. Infrared and Laser Engineering, 42(S2) (2013) 476-780.

[10] W. Gao, Y. Ben and Q. Li. Initial alignment for strapdown inertial navigation system [M]. National Defense Industry Press, Beijing, 2014.

[11] Z. Peng, R. Zhang, M. Guo and L. Liu, etc. Strong tracking filter based SINS initial alignment for large misalignment angles [J]. Journal of Chinese Inertial Technology, 23(5) (2015) 565-569. (in Chinese)

[12] Z. Ding, H. Cai, H. Yang and Y. Cao. Attitude matching for gimbaled inertial navigation system transfer alignment [J]. Mechanics and Materials. 190-191 (2012) 768-773.

[13] Z. Ding, H. Cai and H. Yang. Novel transfer alignment of shipborne gimbaled inertial navigation systems [J]. Trans. Japan Soc. Aero. Space Sci. 57(2) (2014) 93-100. 